\begin{tabular}{|c|}
\hline ARTIGO \\
Recebido em: \\
$11 / 10 / 2013$ \\
\\
Aceito em: \\
$11 / 03 / 2014$ \\
\hline
\end{tabular}

\title{
Enseñanza digital versus derechos de autor: el papel de la biblioteca universitaria en apoyo de profesores y alumnos
} E-learning vs.Copyright: the role of the university library in support of teachers and students

\author{
Enrique MURIEL-TORRADO ${ }^{1}$ \\ J. Carlos FERNÁNDEZ-MOLINA ${ }^{2}$
}

\section{RESUMEN}

El tradicional conflicto entre las actividades de enseñanza y la normativa de derechos de autor se ha visto incrementado y complicado en el entorno digital. Las recientes modificaciones en la legislación no han sido adecuadas, por lo que está muy lejos de satisfacer las necesidades mínimas de alumnos y profesores de la enseñanza digital. La biblioteca universitaria, gracias a su privilegiada posición entre las fuentes de información y los usuarios, puede desarrollar una labor esencial de apoyo a los miembros de la comunidad universitaria para ayudarles a afrontar con éxito la frecuente colisión entre sus intereses y los de los titulares de los derechos sobre las obras intelectuales.

PALABRAS-CLAVE: Derechos de autor. E-learning. Enseñanza. Bibliotecas universitarias. Alfabetización informacional

\begin{abstract}
The conflict between traditional teaching activities and copyright has been increased and complicated in the digital environment. Recent changes in legislation have not been adequate, so it is actually far from meet the basic needs of students and teachers in the digital education. The academic library, thanks to its privileged position of intermediary between information sources and users, can develop an essential work to support members of the university community and help them successfully with the frequent collision between their interests and those of the rights holders of intellectual works.
\end{abstract}

KEYWORDS: Copyright. E-learning. Teaching. Academic libraries. Information Literacy.

v. 19, n. $39,2014$. p. $205-226$

ISSN 1518-2924
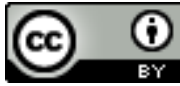

1 Universidade Federal de Santa Catarina - enriquemuriel@gmail.com

2 Universidad de Granada (España) - jcfernan@ugr.es 


\section{INTRODUCCIÓN}

La legislación de derechos de autor tiene una gran influencia en el desarrollo de las actividades de educación digital, ya que suponen casi siempre el uso de obras intelectuales que tienen derechos de autor. Los usos típicos incluyen la reproducción de las obras digitales, su distribución física y, sobre todo, la comunicación y la puesta a disposición del público de tales obras. A este respecto, hay que tener en cuenta que el actual desarrollo tecnológico permite que los materiales educativos preparados por los docentes salgan de los despachos, ordenadores y archivos personales de los profesores y se pongan en línea para ser compartidos por otros miembros de la comunidad educativa. Por tanto, es imprescindible ser consciente de las implicaciones legales que eso supone, es decir, hay que asegurarse de que esos materiales y recursos docentes cumplen con la legislación de derecho de autor, por un lado, y pueden ser protegidos por ella, por otro (HOBBS; JASZI; AUFDERHEIDE, 2007).

Precisamente por el desarrollo de las tecnologías de la información y la comunicación, la legislación de derecho de autor está siendo sometida a una profunda revisión en los últimos años, tanto en los tratados y acuerdos internacionales como en las leyes nacionales. En el ámbito internacional el punto de partida es sin duda el Tratado de Derecho de Autor de la OMPI (1996), que estableció las bases sobre las que se debían sustentar las modificaciones de las legislaciones nacionales para hacer frente a los retos del entorno digital. Si analizamos su contenido nos encontramos con que hay dos aspectos que tienen una gran influencia en la enseñanza digital. El primero es la ampliación del derecho de comunicación al público para incluir la puesta a disposición de la obra de manera que cualquier persona pueda acceder a ella en el momento y lugar que estime oportunos (artículo 8). El segundo es la protección legal de las medidas tecnológicas (sistemas DRM) que protegen las obras con derechos de autor, tales como sistemas anticopia, de control de acceso, etc. (artículo 11) Ambos elementos han sido incluidos en las legislaciones nacionales de todos aquellos países que han modificado su ley recientemente, entre ellos, Brasil (1998) o España (2006). 
Desgraciadamente, las reformas legislativas no han respondido a lo requerido para adaptarse con garantías al nuevo entorno tecnológico. Aunque es habitual y en cierta medida inevitable que la legislación vaya por detrás de la realidad tecnológica, en este caso el desajuste entre legislación y realidad es tan grande que impide que las actividades de enseñanza digital se puedan desarrollar con normalidad. Esto no sólo se debe al lógico retraso de la ley respecto al desarrollo tecnológico, sino también a una fuerte resistencia al cambio por parte de la industria de los derechos de autor. En efecto, los propietarios de derechos de autor y sus lobbys han conseguido en buena medida que las reformas legislativas se hayan quedado cortas y sigan teniendo una importante filosofía pre-digital. Por esa razón, las leyes de derechos de autor actuales no son adecuadas para hacer frente a las necesidades de la enseñanza digital, por lo que constituyen en muchas ocasiones un auténtico obstáculo para el desenvolvimiento de las actividades de enseñanza digital (FISHER; MCGEVERAN, 2006; WALLACE, 2006)

Además de los cambios producidos en la legislación, y en buena medida por su inadecuación, han surgido diversos movimientos internacionales sobre la necesidad de cambiar el actual sistema legal de derechos de autor, y que son conocidos de forma genérica como "copyleft". Entre estos movimientos es especialmente interesante para la enseñanza universitaria el que se centra en la creación de licencias más flexibles que las actuales, en concreto las denominadas "Creative Commons". Este modelo de licencias comenzó a usarse con éxito en los campos de la música y lo audiovisual, pero después ha ido extendiéndose al mundo científico y educativo con resultados también satisfactorios como, por ejemplo, con la iniciativa MIT Open CourseWare.

Dado que los profesores y alumnos pueden ser tanto productores como consumidores de contenidos e-learning, es imprescindible que tengan una clara idea de qué quieren otros que seamos capaces de hacer con el producto de su trabajo y qué queremos nosotros que ellos puedan hacer con el fruto del nuestro. Algo similar sucede con las licencias de tipo copyleft, si se quiere sacar el máximo rendimiento de ellas es necesario tener unos mínimos conocimientos sobre ellas: su tipología, para qué sirve cada una de ellas, cómo pueden 
ayudarnos a compartir nuestros trabajos y utilizar los de los demás, etc. Sin embargo, parece haber una considerable falta de conocimiento y conciencia sobre los problemas de derecho de autor implicados en las actividades de enseñanza digital y sobre las licencias copyleft, tanto entre profesores (FERNÁNDEZ-MOLINA et al., 2011; SMITH et al., 2006) como entre alumnos (FLEGG, 2012; MURIEL-TORRADO, 2012; WU et al., 2010).

Ninguna institución se encuentra en mejor posición que la biblioteca universitaria para ayudar a sus usuarios, profesores y alumnos, a que afronten estos retos con garantía. De hecho, cómo advierte entre otros un estudio de la Association of College and Research Libraries (ACRL, 2010), uno de los nuevos roles de los bibliotecarios de las universidades es precisamente asumir un protagonismo más activo para proporcionar guía, asesoramiento $\mathrm{y}$ concienciación a profesores y estudiantes acerca de los derechos de autor.

El objetivo de este trabajo es presentar los principales puntos de colisión entre las actividades de la enseñanza digital y la legislación de los derechos de autor, junto con el papel que la biblioteca universitaria puede jugar para afrontarlos. Para ello, comenzamos pasando revista a los elementos esenciales de la legislación de derechos de autor y en qué medida afecta a la enseñanza digital, para, a continuación, analizar cómo está cambiando la biblioteca universitaria en los últimos años y cuál debería ser su papel en este conflicto.

\section{ENSEÑANZA DIGITAL VERSUS DERECHOS DE AUTOR}

Los inconvenientes con la legislación de derecho de autor que pueden surgir en el desarrollo de la enseñanza digital son amplios y variados, por lo que resulta útil agruparlos en tres categorías: a) conflictos con cada de uno de los derechos de autor; b) conflictos con la protección tecnológica de las obras (sistemas DRM); c) problemas de permisos y licencias. Como referencia tomaremos las legislaciones de Brasil y España, con bastantes puntos en común. 


\subsection{Conflictos con los derechos de autor}

Tanto Brasil como España tienen un sistema jurídico de tipo latino, por lo que comparten una serie de elementos básicos en sus legislaciones de derecho de autor, entre los que destacan la distinción entre derechos morales y derechos patrimoniales. Los derechos morales son aquellas facultades de carácter personalísimo que tienen los autores de las obras intelectuales. No son de carácter económico, aunque pueden tener consecuencias de esta naturaleza. Los esenciales son el derecho de paternidad, el de integridad y el de divulgación.

El primero de ellos consiste en algo tan simple como el derecho a exigir y reivindicar el reconocimiento como autor de la obra (artículo 24, I de la ley brasileña Brasil y artículo 14.3 de la española). Las implicaciones de este derecho son muy variadas, por ejemplo, debemos tenerlo en cuenta tanto si distribuimos material ajeno (por ejemplo, fragmentos de una obra) a los alumnos, como dentro de nuestro propio material, donde debemos identificar la autoría de los trabajos que utilicemos o referenciemos. También es de gran relevancia el segundo de ellos, el de integridad, que consiste en que los autores pueden exigir que se respete la integridad de la obra, oponiéndose a cualquier deformación, modificación o alteración que perjudique sus legítimos intereses o reputación. Aparece reconocido en el artículo 24, IV de la ley brasileña y en el 14.4 de la española. Entre los ejemplos más habituales podemos mencionar la extracción de un fragmento de una obra descontextualizado, que puede dar a entender un punto de vista contrario al que el autor pretende. Todavía más claro es el caso de tomar una obra y cambiar las partes que consideremos oportunas, alterar el contenido o mutilar alguna de sus fragmentos, de manera que termine siendo irreconocible. El tercero, el de divulgación, consiste en que el autor es el único que puede decidir si divulga su obra y bajo qué forma, nadie puede obligarle a ello. Tanto el artículo 24, III de la ley brasileña como el 14.1 de la española lo reconocen de manera inequívoca. Un ejemplo de conflicto podría ser la distribución sin permiso de documentos internos e inéditos de compañeros de un grupo de trabajo.

Los cuatro principales derechos patrimoniales o económicos también pueden suponer un problema para las actividades educativas. El primero de 
ellos es el clásico derecho de reproducción, cuya esencia es muy simple: al autor le corresponde el derecho a permitir o no la copia o reproducción de su obra por cualquier medio o formato (artículo 29, I de la ley brasileña y 18 de la española). Buena parte de los usos que se realizan sobre las obras protegidas por el derecho de autor, ya sean artículos de revista, libros, fotografías, música, etc., afecta al derecho de reproducción. Desde las clásicas acciones de fotocopiar o microfilmar a las más recientes como digitalizar o escanear. Por supuesto, también la descarga o bajada (download) de materiales de Internet estaría incluido dentro del derecho patrimonial de la reproducción, ya que la actividad que se realiza durante una descarga es una copia, es decir, una reproducción del archivo subido a un servidor accesible a través de la red. Otros supuestos en los que ejercemos la reproducción son: al convertir a formato digital (por ejemplo, a DVD), cintas VHS, o cuando reproducimos un fragmento de una obra ajena en una obra propia, es decir al realizar una cita. Respecto a las copias efímeras o temporales que se producen en los servidores intermediarios de Internet, durante el proceso de transmisión de obras desde un punto a otro, aunque son reproducciones en sentido estricto, están exceptuadas de este derecho si concurren una serie de condiciones (BERCOVITZ et al., 2006).

Muy unido al derecho de reproducción tenemos el segundo de los derechos patrimoniales, el de distribución, que consiste en poner a disposición del público la obra o copias de la misma, en un soporte tangible, mediante venta, alquiler, préstamo, donación, etc. La esencia de este derecho, que aparece reconocido en el artículo 29, VI de la ley brasileña y el 19 de la española, es que siempre se refiere a ejemplares tangibles en cualquier tipo de soporte: analógico (discos, libros o videocasetes), magnético (disquetes), óptico (CD, DVD), electrónico (pendrives, discos duros externos). En ningún caso puede extenderse a obras plasmadas en soportes electrónicos intangibles. Entre los ejemplos que afectan a la enseñanza podemos mencionar el suministro de fotocopias de documentos o el reparto de discos o pendrives que contienen obras a los alumnos. 
El derecho de comunicación al público consiste en que el autor tiene el derecho a autorizar o no los actos por los que su obra está accesible para un conjunto de personas sin previa distribución de ejemplares a cada una de ellas. Este derecho, reconocido en el artículo 29, VII/VIII de la ley brasileña y 20 de la española, es sin duda el de mayor relevancia en el ámbito digital, dado el carácter intangible de la comunicación de la obra. Los supuestos de comunicación pública son muy numerosos, por ejemplo: proyección de películas, vídeos, documentales o cualquier obra audiovisual, exposición de obras de arte, radiodifusión, representaciones escénicas, disertaciones, ejecución de obras musicales, etc. Y si nos centramos en el mundo de la enseñanza, incluiría actividades tan frecuentes como poner información electrónica, fotografías o documentos a disposición de los usuarios en una intranet o Internet, poner contenidos en plataformas de enseñanza, bibliotecas o archivos digitales, tales como tesis, libros electrónicos, artículos, etc., pasar diapositivas, presentaciones digitales o transparencias en clase. Como se ha mencionado previamente, la ampliación del ámbito de este derecho es una de las claves de la modernización de la legislación de derechos de autor. A partir de lo establecido en el artículo 8 del Tratado de la OMPI de 1996, la mayor parte de los países han modificado su ley para que este derecho no solo incluya la comunicación unidireccional, por ejemplo, cuando se proyecta una película que el público ve en un momento y lugar concretos, sino también la denominada puesta a disposición del público, es decir, la obra se coloca en un lugar para que esté accesible a las personas del público en el momento y lugar que ellas elijan.

Finalmente, el cuarto de los derechos de carácter patrimonial es el de transformación, es decir, el de permitir o no la creación de obras derivadas a partir de la obra original. Los casos más clásicos son las traducciones, las adaptaciones y los arreglos musicales, pero incluye cualquier transformación de la obra. Recogido en el artículo 29, III/ IV de la ley brasileña y el 21 de la española, este derecho plantea algunos problemas en el entorno digital, en especial por la facilidad para modificar las obras digitales. Esto supone que las fronteras de este derecho son más difusas, existiendo diversos criterios sobre cuándo se produce o no la transformación. 
Así, mientras para unos cambiar una obra de formato analógico a digital afecta a este derecho, para otros es simplemente una extensión del derecho de reproducción. También resulta dudoso si es una transformación o no el resultado del empleo de un programa de lectura automático sobre una obra electrónica, ya que se podría obtener un archivo de audio de la obra original, resultado que probablemente no estaba previsto por el autor.

Para comprender mejor cómo entran en conflicto estos derechos con las actividades habituales de la enseñanza digital, a continuación incluimos un cuadro en el que se relacionan diversas actividades frecuentes en el entorno educativo con el derecho o derechos afectados.

Cuadro 1. Actividades en la enseñanza y derechos a los que afecta.

\begin{tabular}{|l|l|}
\hline Actividad & Derecho relacionado \\
\hline $\begin{array}{l}\text { Transmisión de sonido o imágenes a través de la } \\
\text { red, videoconferencias, podcasts, etc. }\end{array}$ & Comunicación pública \\
\hline $\begin{array}{l}\text { Descargar obras de una plataforma en línea o } \\
\text { servicios de alojamiento como Dropbox, } \\
\text { Rapidshare, etc. }\end{array}$ & Reproducción \\
\hline $\begin{array}{l}\text { Descargar material de una red p2p como } \\
\text { Bittorrent, Emule, etc. }\end{array}$ & $\begin{array}{l}\text { Reproducción } \\
\text { comunicación pública }\end{array}$ \\
\hline $\begin{array}{l}\text { Envíos por correo electrónico a los alumnos de } \\
\text { clase }\end{array}$ & Comunicación pública \\
\hline $\begin{array}{l}\text { Uso de recursos electrónicos, bases de datos, } \\
\text { publicaciones periódicas, etc. }\end{array}$ & $\begin{array}{l}\text { Según licencias } \\
\text { tienen, igual que otras } \\
\text { obras) }\end{array}$ \\
\hline $\begin{array}{l}\text { Subida a la red de materiales o trabajos de los } \\
\text { alumnos }\end{array}$ & $\begin{array}{l}\text { Reproducción } \\
\text { Comunicación pública }\end{array}$ \\
\hline $\begin{array}{l}\text { Facilitar programas de ordenador propietarios, } \\
\text { shareware, etc. }\end{array}$ & $\begin{array}{l}\text { Licencias (si no tienen, } \\
\text { igual que otras obras) }\end{array}$ \\
\hline $\begin{array}{l}\text { Identificar correctamente el material que } \\
\text { ponemos a disposición de los alumnos }\end{array}$ & Paternidad \\
\hline $\begin{array}{l}\text { Descargar de Internet, digitalizar, imprimir o } \\
\text { copiar un archivo }\end{array}$ & Reproducción \\
\hline $\begin{array}{l}\text { Proyección de películas, documentales, vídeos de } \\
\text { webs como YouTube, Vimeo, etc. }\end{array}$ & Comunicación pública \\
\hline $\begin{array}{l}\text { Pasar un documento de un formato a otro (p.ej. un } \\
\text { archivo de texto a un archivo de audio) }\end{array}$ & Transformación \\
\hline $\begin{array}{l}\text { Tengo un blog de divulgación en el que hablo } \\
\text { sobre mis investigaciones y en ocasiones "cuelgo" } \\
\text { material protegido }\end{array}$ & Comunicación pública \\
\hline
\end{tabular}




\subsection{Protección tecnológica}

La legislación de derechos de autor no es el único obstáculo que nos encontramos cuando queremos utilizar obras con derechos de autor, ya que la protección legal se ha añadido la protección llevada a cabo a través de sistemas DRM (Digital Rights Management), cuyo origen se encuentra en la decisión de los propietarios de los derechos de autor de complementar la protección legal, que consideraban insuficiente en el entorno digital, con la tecnológica. Su razón de ser se encuentra perfectamente definida en la frase de Clark (1996): "the answer to the machine is in the machine", es decir, se trata de una serie de mecanismos que permiten identificar los materiales susceptibles de ser protegidos por los derechos de autor y que controlan el uso que se hace de éstos, evitando pérdidas económicas para quienes ostentan los derechos patrimoniales así como la violación de los derechos morales de sus autores (FERNÁNDEZ-MOLINA, 2003). Sus funciones son variadas: mantenimiento de la integridad impidiendo la modificación de las obras digitales; seguridad en la distribución al registrar autores, productores, editores, etc.; autentificación de los contenidos; control de transacciones; identificación de compradores y vendedores; impedir la descarga por Internet; medidas anticopia, antigrabación o antiregrabación, etc.

Pero estos sistemas no serían de mucha utilidad si no estuvieran protegidos por la legislación de derechos de autor. Y nuevamente el punto de partida es el Tratado de la OMPI, en concreto en su artículo 11, titulado "obligaciones relativas a las medidas tecnológicas", y que establece que hay que proporcionar protección jurídica adecuada y recursos jurídicos efectivos contra la acción de eludir las medidas tecnológicas efectivas que sean utilizadas por los autores en relación con el ejercicio de sus derechos. Es decir, las obras no solo están protegidas por los sistemas DRM, sino que éstos también lo están por la ley. Como advierte Sirinelli (2001), el resultado es una estructura a tres niveles: a) la protección legal (leyes de derecho de autor); b) la protección tecnológica; y

c) las normas legales que protegen las medidas tecnológicas contra su elusión o neutralización. 
Esta nueva protección también ha sido incluida en las leyes nacionales de Brasil y España. De hecho, Brasil fue de los primeros países en hacerlo, ya que en la reforma de 1998 se introdujo el artículo 107, que en sus apartados I y II, prohíbe la alteración, supresión, modificación o inutilización de los dispositivos técnicos utilizados para evitar o restringir la copia o la comunicación pública de las obras. Similar es el caso de la legislación española, aunque algo más tarde, en 2006, cuando se introdujo el artículo 160 para prohibir tanto la elusión individual de las medidas tecnológicas como las denominadas "actividades preparatorias", es decir, el comercio o difusión de dispositivos, productos, componentes o servicios para eludir la protección.

\subsection{Solicitud de permisos y licencias}

Cuando deseamos utilizar una obra protegida por los derechos de autor y no podemos beneficiarnos de ninguna de las limitaciones y excepciones establecidas en la ley, es necesario solicitar permiso al titular de los derechos, una tarea que no siempre resulta sencilla por diferentes razones. De hecho, localizar a los titulares de los derechos de obras no publicadas, agotadas, descatalogadas o de trabajos con una cierta antigüedad puede ser muy complejo. Además, en el medio digital un autor puede diseminar su trabajo sin la intermediación de un editor, por lo que la dificultad es aún mayor. Incluso cabe la posibilidad de que, tras localizar a un titular, nos encontremos con que no tiene todos los derechos, sino solo una parte. Por otra parte, la consulta a los titulares puede llevar meses para obtener respuesta o ni siquiera recibir contestación.

Tras conseguir localizar al titular y recibir una respuesta, es momento de conocer el precio a pagar por el uso de la obra. Las tarifas para usos digitales suelen ser superiores a las relativas para usos analógicos, en ocasiones excesivamente altas para ser afrontadas por los centros educativos. Parece ser que el miedo a lo digital impera entre los titulares, que prefieren aumentar los costes de uso de las obras en el ámbito electrónico, aunque estos usos sean similares a los realizados con documentos físicos. En lugar de apostar por la diseminación de los trabajos parecen inclinarse por tratar de amarrar 
fuertemente las obras que son de su titularidad. A día de hoy no está muy claro el modelo de negocio para los documentos electrónicos digitales, donde también entra en juego la excesiva prevención de los propietarios de derechos -cuando no simple hostilidad- que encarece su precio, lo que provoca un círculo vicioso consistente en una oferta limitada a un alto precio, de manera que se consiguen pocos clientes y, como es lógico, al existir poca demanda la oferta es escasa y cara.

Por otra parte, debemos tener en cuenta que la licencia a adquirir puede tener algunas restricciones. Existen diferencias importantes entre vender una obra en papel y licenciarla para su versión electrónica. La venta de una copia física implica la transferencia total de los derechos de propiedad sobre esa copia de la obra a excepción claro está de los derechos de propiedad intelectual, en cambio, las licencias son contratos (acuerdos privados) que proporcionan normalmente una transferencia limitada de derechos que básicamente consiste en la autorización para acceder a ella. Es decir, una vez adquirida una licencia sobre una obra, no significa que podamos hacer con ella todo lo que consideremos oportuno. A pesar de haber pagado, debemos actuar en función de las instrucciones impuestas por la licencia. También hay que comprobar si la licencia presenta alguna limitación espacial. Existen universidades que tienen inconvenientes con licencias que limitan el acceso sólo a usuarios que están dentro del campus y que obligan a pagar tarifas muy altas para permitir el uso de esos recursos a usuarios que se encuentren fuera de él.

Existe también un tipo de obras que conlleva problemas añadidos, en concreto las denominadas "obras huérfanas". Se trata de obras que aunque todavía están protegidas por el derecho de autor, resulta muy difícil -y en ocasiones imposible- identificar a los autores o titulares de los derechos para poder solicitar una autorización. Esto suele suceder cuando no aparece bien identificado el autor o porque no se consigue localizar a los titulares. La dimensión de este problema es mayor de lo que se podría pensar a simple vista, ya que se calcula que hay alrededor de 3 millones de obras de estas características (VUOPALA, 2010). La localización de los propietarios de los derechos conlleva una gran cantidad de dinero y esfuerzo, y cuando no pueden 
ser localizados, las universidades, bibliotecas, editoriales y museos son reacios al uso del material por miedo a las posibles consecuencias legales si el autor aparece.

\subsection{Posibles soluciones}

Para superar estas dificultades son posibles diversas soluciones, que vamos a analizar en el mismo orden en que han sido expuestas: derechos, protección tecnológica y permisos y licencias.

Si una obra tiene derechos de autor, la única forma de utilizarla sin infringir la ley es acogerse a alguna de las limitaciones y excepciones recogidas en la ley. En el caso del entorno educativo, las más útiles son la ilustración para la enseñanza, los privilegios de las bibliotecas y el derecho de cita. La primera de ellas no estaba incluida en la legislación española hasta la reforma de 2006. En concreto, su artículo 32.2 establece que el profesorado de la educación reglada no necesitará la autorización del titular de los derechos para realizar actos de reproducción, distribución y comunicación pública de pequeños fragmentos de obras o de obras aisladas de carácter plástico o fotográfico figurativo, salvo los libros de texto y los manuales universitarios, y siempre que sea para la ilustración de sus actividades educativas en las aulas, en la medida justificada por la finalidad no comercial perseguida, se trate de obras ya divulgadas y se incluyan el nombre del autor y la fuente. Aunque sin duda es positiva su inclusión en la ley, las múltiples restricciones incluidas en su redacción la alejan mucho de lo deseado: sólo pueden utilizarla los profesores de enseñanza reglada, sólo pequeños fragmentos y excluye los libros de texto y los manuales universitarios. No obstante, lo peor de su contenido es que sólo es aplicable a las actividades realizadas "en las aulas", por lo que deja fuera toda la enseñanza digital. Respecto a las excepciones a favor de bibliotecas e instituciones similares, se incluyen habitualmente en las legislaciones de derechos de autor porque se considera que sus funciones de difusión y preservación de la información son de interés público. 
En el caso de España, el artículo 37 de su ley les permite hacer reproducciones de las obras por razones de investigación y preservación, lo que sin duda contribuye a facilitar las actividades educativas. Sin embargo, el nuevo apartado 3 incluido tras la reforma de 2006, y dedicado al derecho de comunicación pública, será de muy escasa utilidad. Permite los actos de comunicación o puesta a disposición del público de las obras que componen su colección, siempre que sea a través de terminales especializados situados en la biblioteca, pero, y aquí está el problema, siempre que "las obras no estén bajo licencia". Dado que la práctica totalidad de las obras digitales se adquieren bajo licencia, es evidente su escaso interés para facilitar las tareas de profesores y alumnos. Por último, el derecho de cita es una de las limitaciones básicas a los derechos de autor, y de hecho, es la única obligatoria según el Convenio de Berna. Consiste en que en una obra es posible hacer citas de una obra ajena, siempre que ésta se haya hecho lícitamente accesible al público (es decir, se ha divulgado), que se efectúe conforme "a los usos honrados" y "en la medida justificada por el fin que se persiga". Además, las citas deberán mencionar "la fuente y el nombre del autor si ese nombre figura en la fuente". En estos términos, aproximadamente, es regulada por el artículo 32.1 de la ley española. Muy similar es la ley brasileña, cuyo artículo 46, III permite la cita en todo tipo de obras de pasajes de cualquier otra obra, siempre que sea para fines de estudio, crítica o polémica, en la medida necesario para cumplir dicho fin, e indicando el nombre del autor y el origen de la obra. Sin embargo, la ley brasileña no recoge ni la limitación de ilustración para la enseñanza ni los privilegios de bibliotecas, por lo que las universidades tienen serias dificultades para llevar a cabo sus actividades sin infringir la ley de derechos de autor.

En cuanto a los problemas causados por la protección tecnológica, se ha intentado -aunque con escaso éxito, como veremos a continuación- introducir medidas de corrección. En concreto, el artículo 161 de la ley española obliga a que los titulares de los derechos sobre las obras faciliten su disfrute a los beneficiarios de las limitaciones y excepciones, incluidas las que acabamos de mencionar. Sin embargo, si los titulares no toman voluntariamente las medidas adecuadas para ello, a los que pretenden beneficiarse de las limitaciones solo les 
queda la opción de recurrir a los tribunales ordinarios, lo que resulta muy poco viable. Por si esto fuera poco, esta medida no es aplicable a las obras que se han puesto a disposición del público bajo licencia, que como ya hemos mencionado, son la práctica totalidad de las obras digitales. En definitiva, la protección tecnológica supone un obstáculo casi insalvable para utilizar las obras aunque sea para usos permitidos. El caso de la ley brasileña es incluso peor, ya que su ley ni siquiera regula cuál es la relación entre estas medidas y las limitaciones y excepciones a los derechos de autor.

Finalmente, respecto a los problemas con los permisos, una buena solución son las licencias colectivas. Permiten la utilización de una serie de obras que están dentro de un catálogo que generalmente abarca un amplio rango de obras, de manera que no es necesario tener que negociar licencia a licencia los trabajos que queramos emplear. La gestión corre a cargo de las sociedades de gestión colectiva de los derechos, como CEDRO en España, Copyright Licensing Agency en el Reino Unido o Copyright Clearance Center en Estados Unidos. Además, para afrontar el problema de que los derechos pertenezcan titulares de diferentes países, se han puesto en marcha diferentes acuerdos e iniciativas que van más allá de las fronteras nacionales, por ejemplo, el Repertorio Integrado de las Entidades de Derechos Reprográficos de Iberoamérica (RIEDRI). Por lo que se refiere al problema de las obras huérfanas, han surgido varias iniciativas en los últimos años intentando solucionarlo, en especial en Estados Unidos y en Europa. En el caso estadounidense se llevó a cabo un estudio (COPYRIGHT OFFICE, 2006), cuya idea central es que se establezca un sistema de limitación de responsabilidad para aquellos usuarios capaces de demostrar haber realizado una "búsqueda razonablemente diligente" del titular de los derechos. En este caso, el usuario podrá utilizar la obra huérfana y sólo deberá compensar "razonablemente" al titular en el caso de que éste reclame. Aunque ha habido varias iniciativas legislativas al respecto, ninguna se ha convertido en ley hasta ahora. En cuanto a la Unión Europea, también elaboró un informe (COPYRIGHT SUBGROUP, 2007) y recientemente aprobó una directiva (UNIÓN EUROPEA, 2012), cuya transposición debería modificar las leyes nacionales de los países europeos en poco tiempo. También 
en el contexto europeo, vale la pena reseñar el proyecto Arrow (http://www.arrow-net.eu), cuyo objetivo es ofrecer otras formas de aclarar el estado de los derechos de obras huérfanas y descatalogadas para que puedan ser digitalizadas e incluidas en la biblioteca digital europea (Europeana).

Finalmente, no podemos dejar de reseñar lo útiles que pueden ser en este contexto las licencias de tipo copyleft, por ejemplo las Creative Commons. Proporcionan un sistema muy flexible y simple para que el autor conceda unos derechos y se reserve otros, facilitando que todos los usuarios, personales e institucionales, sepan qué se puede o no hacer con la obra. Su utilización por parte de iniciativas de tanto éxito como OpenCourseWare es un buen ejemplo de su adecuación para facilitar las actividades de enseñanza digital.

\section{PAPEL DE LA BIBLIOTECA UNIVERSITARIA}

En los últimos años, la adecuación y adaptación de las bibliotecas universitarias a la realidad digital han sido seriamente cuestionadas. Oakleaf (2010) recoge algunas de las principales críticas recibidas, entre las que podemos destacar el rol pasivo de los bibliotecarios en el campus, que tal vez no han sabido explicar adecuadamente lo que hacían, la desafortunada imagen que en ocasiones han proyectado como meros guardianes de libros, la percepción de la biblioteca como una organización aislada del resto del campus, sin participación dentro de las decisiones estratégicas, o la incapacidad para demostrar la contribución de sus servicios a la comunidad universitaria.

Para hacer frente al nuevo entorno tecnológico y educativo se han hecho algunas propuestas, cuyo objetivo básico es ampliar la visión tradicional de la biblioteca como el lugar donde se asienta la colección (ATTIS, 2013), expandiendo sus servicios en cuatro diferentes áreas: la biblioteca como lugar físico donde se ofrecen servicios, estudio colaborativo, tutorías, cafetería, etcétera; apoyo a la investigación, orientación en cuestiones ético-jurídicas, apoyo a la publicación o curación de datos; servicios web, búsquedas, bases de datos o acceso móvil; y, por último, apoyo al aprendizaje, entre otros, en 
producción multimedia, diseño de planes de estudio y alfabetización informacional.

Otro importante cambio se ha producido respecto a la fondos, olvidándose del imposible objetivo de tener una colección que lo abarque todo, y apostando por desarrollarla de forma estratégica bajo un modelo orientado en lugar de en la tradicional posesión del material- al acceso a las obras para el presente y el futuro (HARVARD UNIVERSITY, 2009). En esta línea, las bibliotecas han realizado grandes inversiones de recursos en servicios basados en la web (KIM, 2011), pagando un alto precio por el acceso a bases de datos y catálogos editoriales, mientras que por otro lado también se procura potenciar el acceso abierto de la producción científica propia para el repositorio institucional. Esta inversión en recursos digitales debería complementarse, por un lado, con el fomento de su uso y, por otro, con la necesaria formación de los usuarios, particularmente en el aspecto de los derechos de autor, de forma que los interesados conozcan cómo utilizar el material, cuáles son las condiciones de uso de las bases de datos o qué derechos tienen como usuarios y como creadores también de obras.

Lógicamente, el papel del bibliotecario universitario también ha evolucionado incansablemente en los últimos años y, como señalan Alsop y Bordonaro (2007), se han mostrado abiertos a expandir su rol en el campus, redefiniendo así una vez más una profesión en continua evolución que debe adaptarse permanentemente al presente para continuar siendo relevante en el futuro. Y uno de los nuevos roles que mayor relevancia está alcanzando, como señala el reciente informe de la ACRL (2013), es precisamente la buena comprensión de los derechos de autor y la comunicación científica. En efecto, los bibliotecarios se perfilan como unos buenos comunicadores de información relacionada con los derechos de autor porque interactúan verticalmente con todos los estamentos de la institución académica, desde la administración hasta los estudiantes. A este respecto, como asevera Bay (2001), los bibliotecarios no pueden adoptar un rol pasivo o conservador para evitar conflictos con la legislación, lo que puede provocar que se desista de llevar a cabo actividades que ampara la ley, sino que deben tomar una actitud diligente en defensa de los 
intereses de los usuarios.

El país donde más rápidamente se ha desarrollado la nueva figura del “copyright librarian” es Estados Unidos, probablemente por el impulso que supuso la TEACH Act (Technology, Education and Copyright Harmonization Act de 2002), que obligaba a los centros académicos a designar un responsable "infringement agent" que esté accesible por correo electrónico y pueda responder a reclamaciones de infracciones sobre derechos de autor en un determinado plazo, cometido que ha sido asumido habitualmente por uno de los bibliotecarios de las universidades. Pero además, en los últimos años esta necesidad se ha incrementado (ACRL, 2010) por la aparición de nuevos factores, entre los que destacan el enorme crecimiento y desarrollo de las actividades de enseñanza online, cuyo ejemplo más significativo son los MOOC (Cursos en Línea Masivos y Abiertos), donde el contenido de los cursos van más allá del ámbito de la institución (ARL, 2013).

Sería muy conveniente que otros países, incluidos España o Brasil, siguieran esta tendencia y fueran creando en sus universidades esta nueva figura profesional, con capacidad para ayudar a resolver las dudas básicas de los usuarios sobre los problemas de derechos de autor que les afectan, o de forma más general, sobre todas las cuestiones jurídicas relacionadas con la comunicación científica, incluido el movimiento Open Access o las licencias Creative Commons. A continuación vamos a comentar brevemente las principales tareas que deberían asumir este tipo de profesionales.

La primera de ellas es la creación de contenidos que ayuden a los usuarios a solucionar las dudas más frecuentes, algo que se puede realizar mediante diferentes perfiles de usuarios en función de sus necesidades y recopilando las cuestiones observadas que más se repiten entre ellos. Con este punto de partida se puede elaborar una guía básica y permanentemente accesible. Como complemento tendríamos la formación de los usuarios, tanto profesores como estudiantes, no en vano uno de los estándares de alfabetización informacional está centrado en estas cuestiones. Para ello es imprescindible estar en comunicación permanente y proactiva con todas las 
áreas de la institución, en especial con la dirección y, en su caso, el departamento jurídico, de manera que se maximice el rendimiento académico pero respetando la ley, como advierte Vesely (2007). En la misma línea tendríamos la colaboración para combatir el cada vez más presente problema del plagio (SPRING; ADAMS, 2013), que se produce no solo voluntariamente, para ahorrar tiempo u obtener alguna ventaja, sino también en ocasiones de manera inadvertida o por simple desconocimiento. Finalmente, resulta imprescindible en la situación actual tomar una posición proactiva en todo lo relativo al movimiento copyleft, incluyendo el acceso abierto a la información científica y los repositorios digitales, o las posibilidades de las licencias flexibles de tipo Creative Commons.

Para lograr todas estas tareas con éxito es imprescindible que el bibliotecario esté adecuadamente preparado. Esto supone, por un lado, poseer la capacidad para establecer la comunicación apropiada con sus usuarios, y por otro, tener la suficiente formación sobre cuestiones legales como para poder resolver las dudas y conflictos que surjan, y aprovechar las posibilidades que ofrece la legislación en beneficio de la institución y sus usuarios. Evidentemente, no se trata de que el profesional tenga respuesta para todo, pero al menos debe tener la capacidad para reconocer cuándo sus conocimientos no son suficientes y hay que recurrir a un tercero con la formación adecuada.

\section{CONCLUSIONES}

En los últimos años, los derechos de autor se han convertido en uno de los principales obstáculos para el desarrollo adecuado de las actividades de enseñanza digital en las universidades.

Esto se debe, por un lado, al reforzamiento de los derechos que se ha producido a partir de 1996 en las reformas de las diferentes legislaciones nacionales, tanto por el incremento de su ámbito de aplicación, en especial del derecho de comunicación al público, como por la incorporación de las medidas 
de protección tecnológica (sistemas DRM), que suponen una capa más de protección para las obras; y por otro, a que dichas reformas legales no han sido equilibradas, ya que el aumento de los derechos no ha ido acompañado de la correspondiente adaptación y ampliación de las excepciones y limitaciones al nuevo entorno digital, quedando éstas relegadas a las obras en formato analógico. Como consecuencia de esta falta de adecuación de la legislación a la realidad tecnológica y educativa, muchos profesores y alumnos se sienten perdidos a la hora de desarrollar sus actividades docentes y discentes, sin saber bien qué pueden o no hacer con las obras de otros ni qué derechos tienen sobre las que ellos crean.

Conscientes de estas dificultades y de la necesidad de ayuda que sus usuarios tienen en estos temas, las bibliotecas universitarias han comenzado a asumir este papel de formación y asesoramiento en cuestiones ético-jurídicas, dando lugar a nuevos servicios y roles profesionales. De hecho, cada vez es más frecuente que haya bibliotecas universitarias que tienen un departamento de asesoría en derechos de autor, en especial en Estados Unidos, sin duda los pioneros en esta tendencia.

Para que estas iniciativas tengan éxito deben concurrir al menos dos condiciones. La primera es que los bibliotecarios de las universidades sean conscientes de que deben aprovechar su tradicional posición de intermediarios entre la información y los usuarios para ofrecerles apoyo y ayuda en este tipo de problemas, no dejándolo en manos de otros tipos de profesionales (gabinete jurídico, por ejemplo) ni permitiendo que sean los propios usuarios los que intenten resolverlas recurriendo a fuentes de escasa fiabilidad en Internet. La segunda es que deben tener la formación adecuada para poder ayudar y asesorar a los usuarios en estas cuestiones ético-jurídicas. A día de hoy parece más fácil cumplir la primera de las condiciones, ya que hay numerosos ejemplos de iniciativas que ponen de manifiesto esa creciente concienciación; sin embargo, los resultados de los pocos estudios realizados hasta ahora sobre la materia demuestran que la mayor parte de los bibliotecarios carecen de los conocimientos mínimos exigibles para desarrollar con garantía tal labor de asesoramiento y formación. 


\section{BIBLIOGRAFÍA}

ACRL. 2010 Top Ten Trends in Academic Libraries: A Review of the Current Literature. College and Research Libraries News, v. 7, n. 6, p. 286-292, 2010. ACRL. Intersections of Creating Strategic Collaborations for a Changing Academic Environment Scholarly Communication and Information Literacy. Chicago: . Chicago: Association of College and Research Libraries, 2013.

ALSOP, J.; BORDONARO, K. Multiple Roles of Academic Librarians. Electronic Journal of Academic and Special Librarianship, v. 8, n. 1, 2007.

ARL. New Roles for New Times : Transforming Liaison Roles in Research Libraries. [s.l: s.n.].

ATTIS, D. Redefining the Academic Library: Managing the Migration to Digital Information Services. [s.l: s.n.]. Disponível em:

$<$ http://digitalcommons.mcmaster.ca/cgi/viewcontent.cgi?article=1000\&conte xt=lookingforward $>$. Acesso em: 10 fev. 2014.

BAY, M. T. Libraries and the Need to Educate Users about Copyright and Fair Use. Library Hi Tech News, v. 18, n. 5, 5 jan. 2001.

BERCOVITZ, R. et al. Las reformas de la Ley de Propiedad Intelectual.

Valencia: [s.n.].

BRASIL. LEI No 9.610, DE 19 DE FEVEREIRO DE 1998. Altera, atualiza e consolida a legislação sobre direitos autorais e dá outras providências., 1998. Disponível em: <http://www.planalto.gov.br/ccivil_03/LEIS/L9610.htm> CLARK, C. The answer to the machine is in the machine (P. B. HUGENHOLTZ, Ed.)The Future of Copyright in a Digital Environment: Proceedings of the Royal Academy Colloquium Organized by the Royal Netherlands Academy of Sciences (KNAW) and the Institute for Information Law. Anais...Amsterdam: The Hague [et al.]:Kluwer Law International, 1996

COPYRIGHT OFFICE. Report on orphan works. [s.l: s.n.]. Disponível em: <http://www.copyright.gov/orphan/orphan-report-full.pdf>. COPYRIGHT SUBGROUP. Report on Digital Preservation, Orphan Works, and Out-of-Print Works. Selected Implementation Issues. 2007.

ESPAÑA. Ley 23/2006, de 7 de julio, por la que se modifica el texto refundido de la Ley de Propiedad Intelectual, aprobado por el Real Decreto Legislativo 1/1996, de 12 de abril., 2006. Disponível em: <https://www.boe.es/diario_boe/txt.php?id=BOE-A-2006-12308> FERNÁNDEZ-MOLINA, J. et al. Copyright and e-learning: professors' level of knowledge about the new Spanish law. Aslib Proceedings, v. 63, n. 4, p. 340353, 2011.

FERNÁNDEZ-MOLINA, J. C. Laws against the circumvention of copyright technological protection. Journal of Documentation, v. 59, n. 1, p. 41-68, 2003. FISHER, W. W.; MCGEVERAN, W. The Digital Learning Challenge: Obstacles to Educational Uses of Copyrighted Material in the Digital Age. Berkman Center Research Publication, n. 9, 2006.

FLEGG, C. Alumni, Libraries and Universities: whereto the relationship. The Journal of Academic Librarianship, v. 38, n. 1, p. 60-62, jan. 2012. 
HARVARD UNIVERSITY. Report of the Task Force on University Libraries. [s.l: s.n.]. Disponível em:

<http://www.provost.harvard.edu/reports/Library_Task_Force_Report.pdf>. HOBBS, R.; JASZI, P.; AUFDERHEIDE, P. The Cost of Copyright Confusion for Media LiteracyOnline Submission. [s.l: s.n.]. Disponível em:

<http://files.eric.ed.gov/fulltext/ED499465.pdf>. Acesso em: 28 fev. 2014.

KIM, Y.-M. Why Should I Use University Library Website Resources? Discipline Differences. The Journal of Academic Librarianship, v. 37, n. 1, p. 9-18, jan. 2011.

MURIEL-TORRADO, E. Los derechos de autor y la enseñanza en la universidad : el papel de la biblioteca universitaria. Granada: Universidad de Granada, 2012, 479 f. [Tesis doctoral - Facultad de Comunicación y Documentación, Departamento de Información y Comunicación].

OAKLEAF, M. The value of academic libraries: A comprehensive research review and report. [s.l: s.n.]. Disponível em:

<http://www.citeulike.org/group/4576/article/7847697>. Acesso em: $10 \mathrm{fev}$. 2014.

OMPI. Tratado de la OMPI sobre Derecho de Autor. Disponível em:

<http://www.wipo.int/treaties/es/text.jsp?file_id=295167>. Acesso em: 28 fev. 2014.

SIRINELLI, P. The scope of the prohibition on circumvention of technological measures: exceptions. ALAI 2001 Congress, 2001.

SMITH, K. H. et al. Copyright Knowledge of Faculty at Two Academic Health Science Campuses: Results of a Survey. Serials Review, v. 32, n. 2, p. 59-67, jun. 2006.

SPRING, H.; ADAMS, R. Combating plagiarism: the role of the health librarian. Health information and libraries journal, v. 30, n. 4, p. 337-42, dez. 2013. UNIÓN EUROPEA. DIRECTIVA 2012/28/UE DEL PARLAMENTO EUROPEO Y DEL CONSEJO de 25 de octubre de 2012 sobre ciertos usos autorizados de las obras huérfanaswipo.int, 2012. Disponível em:

<http://www.wipo.int/edocs/lexdocs/laws/es/eu/eu169es.pdf>. Acesso em: 28 fev. 2014

VESELY, S. A. Do You Need a Copyright Librarian? Internet Reference Services Quarterly, v. 11, n. 4, p. 69-82, 7 mar. 2007.

VUOPALA, A. Assessment of the orphan works issue and costs for rights clearancef. [s.l: s.n.]. Disponível em: <http://cultivate-cier.nl/wpcontent/uploads/2012/03/vuopala_report.pdf>. Acesso em: 28 fev. 2014. WALLACE, M. An investigation into copyright concerns as a barrier to the widespread development of e-learning practice within scottish further education colleges. Information \& Communications Technology Law, v. 15, n. 1, p. 79-119, mar. 2006.

WU, H.-C. et al. College students' misunderstandings about copyright laws for digital library resources. The Electronic Library, v. 28, n. 2, p. 197-209, 2010. 
